\title{
NNLO results for heavy quark and direct photon production near threshold
}

\author{
Nikolaos Kidonakis ${ }^{\text {a }}$ *

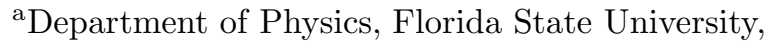 \\ Tallahassee, FL 32306-4350, USA
}

I present some results for the next-to-next-to-leading order expansions of the resummed cross sections for heavy quark and direct photon production near threshold in hadronic collisions.

\section{INTRODUCTION}

Heavy quark and direct photon production in hadronic collisions are processes of great interest. The discovery of the top has increased efforts in the calculation of heavy quark production cross sections while direct photon production is important for determinations of gluon distributions. Near threshold for the production of the final state in these processes one can resum logarithmic corrections originating from soft gluon emission. A formalism and methods for the resummation of soft-gluon contributions at next-to-leading logarithmic (NLL) accuracy for a variety of QCD hard scattering processes, including heavy quark, jet, and direct photon production, have been recently developed (for a review see Ref. [1]). The resummed cross sections have been expanded at next-to-next-to-leading order (NNLO) and exhibit a substantially reduced scale dependence relative to next-to-leading order (NLO).

\section{NNLO RESULTS FOR HEAVY QUARK PRODUCTION}

The heavy quark production cross section in hadronic collisions may be written as a convolution of parton distribution functions $\phi$ with the partonic hard scattering $\hat{\sigma}$ :

$\sigma_{h_{1} h_{2} \rightarrow Q \bar{Q}}=\sum_{f} \phi_{f / h_{1}} \otimes \phi_{\bar{f} / h_{2}} \otimes \hat{\sigma}_{f \bar{f} \rightarrow Q \bar{Q}}$.

\footnotetext{
${ }^{*}$ This work was supported in part by the U.S. Department
} of Energy.
We now define variables $z \equiv Q^{2} / s$ and $\tau \equiv z_{\min }=$ $Q^{2} / S$, where $Q^{2}, s$ and $S$ are the invariant masses squared of the heavy quark pair, and the incoming partons and hadrons, respectively. Then, near threshold, $z=1, \hat{\sigma}$ includes logarithmic terms of the form $-\left(\alpha_{s}{ }^{n} / n !\right)\left[\left(\ln ^{m}(1-z)\right) /(1-z)\right]_{+}$, with $m \leq 2 n-1$ at $n$th order in $\alpha_{s}$, which can be resummed to all orders in perturbative QCD.

If we take moments of Eq. (1), the convolution becomes a simple product. Replacing the incoming hadrons by partons we can then write moments of the partonic cross section as

$$
\begin{array}{r}
\tilde{\sigma}_{f \bar{f} \rightarrow Q \bar{Q}}(N)=\tilde{\phi}_{f / f}(N) \tilde{\phi}_{\bar{f} / \bar{f}}(N) \hat{\sigma}_{f \bar{f} \rightarrow Q \bar{Q}}(N) \\
=\tilde{\psi}_{f / f}(N) \tilde{\psi}_{\bar{f} / \bar{f}}(N) H_{I J} \tilde{S}_{J I}(N),
\end{array}
$$

where the moments are defined by [2] $\tilde{\sigma}(N)=$ $\int_{0}^{1} d \tau \tau^{N-1} \sigma(\tau), \quad \hat{\sigma}(N)=\int_{0}^{1} d z z^{N-1} \hat{\sigma}(z)$, and $\tilde{\phi}(N)=\int_{0}^{1} d x x^{N-1} \phi(x)$. Under moments the plus distributions in $1-z$ produce powers of $\ln N$. In the second line of Eq. (2) we have introduced a refactorization [2] in terms of center-of-mass parton distributions $\psi$, a soft gluon function $S$ which describes noncollinear soft gluons, and the $N$-independent hard scattering $H$. The indices $I, J$ describe color exchange and the functions $H$ and $S$ are matrices in color space.

Using Eq. (2), we then have

$\hat{\sigma}_{f \bar{f} \rightarrow Q \bar{Q}}(N)=\left[\frac{\tilde{\psi}_{f / f}(N)}{\tilde{\phi}_{f / f}(N)}\right]^{2} H_{I J} \tilde{S}_{J I}(N)$.

Resummation follows from the renormalization properties of these functions as described in Refs. 
[1, 2]. The resummed heavy quark cross section in moment space is then

$$
\begin{aligned}
& \hat{\sigma}_{f \bar{f} \rightarrow Q \bar{Q}}(N)=\exp \left\{2\left[E^{(f)}(N)+E_{\text {scale }}^{(f)}\right]\right\} \\
& \times H_{I J}\left(\frac{Q}{\mu}, \alpha_{s}\left(\mu^{2}\right)\right) \tilde{S}_{J I}\left(1, \alpha_{s}\left(Q^{2} / N^{2}\right)\right) \\
& \times \exp \left[\int_{\mu}^{Q / N} \frac{d \bar{\mu}}{\bar{\mu}}\left[\lambda_{I}\left(\alpha_{s}\left(\bar{\mu}^{2}\right)\right)+\lambda_{J}^{*}\left(\alpha_{s}\left(\bar{\mu}^{2}\right)\right)\right]\right] .
\end{aligned}
$$

The function $E^{(f)}$ resums the $N$-dependence of the ratio $\psi_{f / f} / \phi_{f / f}$ [3, 2] while the scale variation is given by $E_{\text {scale }}^{(f)}$. In the last exponent, the $\lambda^{\prime} s$ are eigenvalues of the soft anomalous dimension matrix $\Gamma_{S}$, which is determined from renormalization group analysis of the soft function $S$ and has been calculated explicitly at one-loop in Ref. [2]. For finite-order expansions one does not need to diagonalize $\Gamma_{S}$; this is a simplification compared to the full resummed cross section [4]. Explicit results for all the exponents in the above expression are given in Refs. [1, 2].

The NLO expansions for both the $q \bar{q} \rightarrow Q \bar{Q}$ and $g g \rightarrow Q \bar{Q}$ partonic channels have been obtained in Refs. [1], 2]. These results are in agreement with the one-loop results in Ref. [5].

The expansion of the resummed cross section at NNLO provides us with the dominant terms near threshold at that order. For the $q \bar{q} \rightarrow Q \bar{Q}$ channel in the $\overline{\mathrm{MS}}$ scheme, we find the following NNLO corrections in pair inclusive kinematics:

$$
\begin{aligned}
& \hat{\sigma}_{q \bar{q} \rightarrow Q \bar{Q}}^{\overline{\mathrm{MS}}(2)}\left(1-z, m^{2}, s, t_{1}, u_{1}\right)=\sigma_{q \bar{q} \rightarrow Q \bar{Q}}^{B} \frac{\alpha_{s}^{2}}{\pi^{2}} \\
& \times\left\{8 C_{F}^{2}\left[\frac{\ln ^{3}(1-z)}{1-z}\right]_{+}+\left[\frac{\ln ^{2}(1-z)}{1-z}\right]_{+} C_{F}\left[-\beta_{0}\right.\right. \\
& +12 C_{F}\left(4 \ln \left(\frac{u_{1}}{t_{1}}\right)-\operatorname{Re} L_{\beta}-1-\ln \left(\frac{\mu^{2}}{s}\right)\right) \\
& \left.\left.+6 C_{A}\left(-3 \ln \left(\frac{u_{1}}{t_{1}}\right)-\ln \left(\frac{m^{2} s}{t_{1} u_{1}}\right)+\operatorname{Re} L_{\beta}\right)\right]\right\} \\
& +\cdots
\end{aligned}
$$

where $\sigma_{q \bar{q} \rightarrow Q \bar{Q}}^{B}$ is the Born cross section, $m$ is the heavy quark mass, $t_{1}=\left(p_{q}-p_{\bar{Q}}\right)^{2}-m^{2}$, $u_{1}=\left(p_{\bar{q}}-p_{\bar{Q}}\right)^{2}-m^{2}, \beta_{0}=11-2 n_{f} / 3$, with $n_{f}$ the number of flavors, and we have omitted subleading powers of $\ln (1-z)$.

Analogous results have been obtained in the DIS scheme, and also in single-particle inclusive kinematics in both schemes [6]. The NNLO corrections for the $g g \rightarrow Q \bar{Q}$ channel are lengthier and are presented in Refs. [ 1, 6]. These results are useful not only for the total cross section but also for the calculation of heavy quark differential distributions [7].

\section{NNLO RESULTS FOR DIRECT PHO- TON PRODUCTION}

Next, we discuss direct photon production in hadronic collisions. The factorized cross section may again be written as a convolution of parton distributions with the hard scattering

$\sigma_{h_{1} h_{2} \rightarrow \gamma}=\sum_{f} \phi_{f_{1} / h_{1}} \otimes \phi_{f_{2} / h_{2}} \otimes \hat{\sigma}_{f_{1} f_{2} \rightarrow \gamma}$.

If we take moments of the above equation and replace the incoming hadrons by partons, we can write moments of the partonic cross section as

$$
\begin{array}{r}
\tilde{\sigma}_{f_{1} f_{2} \rightarrow \gamma}(N)=\tilde{\phi}_{f_{1} / f_{1}}(N) \tilde{\phi}_{f_{2} / f_{2}}(N) \hat{\sigma}_{f_{1} f_{2} \rightarrow \gamma}(N) \\
=\tilde{\psi}_{f_{1} / f_{1}}(N) \tilde{\psi}_{f_{2} / f_{2}}(N) \tilde{J}(N) H \tilde{S}(N), \quad(7)
\end{array}
$$

where in the second line of Eq. (7) we have introduced a refactorization as described for heavy quark production. Here, we have in addition a function $J$ which describes the outgoing jet [ 8, 9].

After solving for $\hat{\sigma}_{f_{1} f_{2} \rightarrow \gamma}(N)$ in Eq. (7) and resumming the $N$-dependence of all functions, we may write the resummed cross section as

$$
\begin{aligned}
& \hat{\sigma}_{f_{1} f_{2} \rightarrow \gamma}(N)=\exp \left\{\sum_{i=1,2}\left[E^{\left(f_{i}\right)}\left(N_{i}\right)+E_{\text {scale }}^{\left(f_{i}\right)}\right]\right\} \\
& \times \exp \left\{E_{\left(f_{J}\right)}^{\prime}(N)\right\} H\left(\alpha_{s}\left(\mu^{2}\right)\right) S\left(1, \alpha_{s}\left(S / N^{2}\right)\right) \\
& \left.\times \exp \left[\int_{\mu}^{\sqrt{S} / N} \frac{d \mu^{\prime}}{\mu^{\prime}} 2 \operatorname{Re} \Gamma_{S}\left(\alpha_{s}\left(\mu^{\prime 2}\right)\right)\right]\right\}
\end{aligned}
$$

The exponent $E_{\left(f_{J}\right)}^{\prime}$ resums the $N$-dependence of the outgoing jet [8, 9] while $E^{\left(f_{i}\right)}$ resums the ratio $\psi / \phi$ as we discussed in the previous section. We note that because of the simpler color structure of 


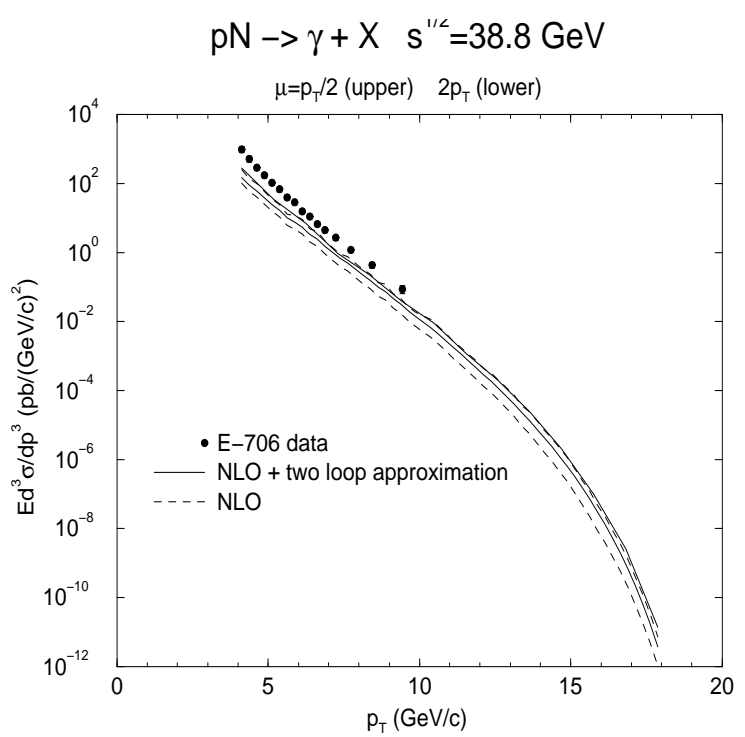

Figure 1. NLO and NNLO results for direct photon production in hadronic collisions.

the hard scattering, here $H, S$, and $\Gamma_{S}$ are simple functions; thus we drop the color indices.

The threshold region is given in terms of the variable $w \equiv-u /(s+t)$ by $w=1$. We also define $v \equiv 1+t / s$ with $s=\left(p_{1}+p_{2}\right)^{2}, t=\left(p_{1}-p_{\gamma}\right)^{2}$, and $u=\left(p_{2}-p_{\gamma}\right)^{2}$. The NLO $\overline{\mathrm{MS}}$ expansion of the resummed cross section is presented in Refs. [9. 10]. Agreement is found with the exact NLO cross section in Ref. [11].

The NNLO $\overline{\mathrm{MS}}$ corrections for the partonic subprocess $q\left(p_{1}\right)+g\left(p_{2}\right) \rightarrow q\left(p_{J}\right)+\gamma\left(p_{\gamma}\right)$ are

$$
\begin{aligned}
& \hat{\sigma}_{q g \rightarrow q \gamma}^{\overline{\mathrm{MS}}(2)}(1-w, s, v)=\sigma_{q g \rightarrow q \gamma}^{B} \frac{\alpha_{s}^{2}}{\pi^{2}} \\
& \times\left\{\left(\frac{C_{F}^{2}}{2}+2 C_{F} C_{A}+2 C_{A}^{2}\right)\left[\frac{\ln ^{3}(1-w)}{1-w}\right]_{+}\right. \\
& +\left[\frac{3}{2} C_{F}^{2}\left(-\frac{3}{4}+\ln v-\ln \left(\frac{\mu^{2}}{s}\right)\right)\right. \\
& \quad+3 C_{A}^{2}\left(\ln \left(\frac{v}{1-v}\right)-\ln \left(\frac{\mu^{2}}{s}\right)\right) \\
& \quad+\frac{3}{2} C_{F} C_{A}\left(-\ln (1-v)+3 \ln v-3 \ln \left(\frac{\mu^{2}}{s}\right)\right. \\
& \left.\left.\left.\quad-\frac{3}{2}\right)-\frac{\beta_{0}}{2}\left(\frac{C_{F}}{4}+C_{A}\right)\right]\left[\frac{\ln ^{2}(1-w)}{1-w}\right]_{+}\right\}
\end{aligned}
$$

$+\cdots$

where $\sigma_{q g \rightarrow q \gamma}^{B}$ is the Born cross section for this channel and we have omitted subleading powers of $\ln (1-w)$. Analogous results have been obtained for the partonic channel $q\left(p_{1}\right)+\bar{q}\left(p_{2}\right) \rightarrow$ $g\left(p_{J}\right)+\gamma\left(p_{\gamma}\right)[1$, 10].

In Fig. 1 we show some numerical results [10 for direct photon production and compare with the experimental results from the E706 Collaboration at Fermilab [12]. We see that the sum of the exact NLO cross section and NNLO approximate corrections shows a much reduced dependence on the factorization scale relative to the exact NLO cross section alone. However, the NNLO cross section is still below the E706 data.

\section{REFERENCES}

1. N. Kidonakis, hep-ph/9902484, submitted to Int. J. Mod. Phys. A; in JHEP Proceedings, Corfu Summer Institute on Elementary Paricle Physics, 1998, hep-ph/9904507.

2. N. Kidonakis and G. Sterman, Phys. Lett. B 387 (1996) 867; Nucl. Phys. B505 (1997) 321; N. Kidonakis, Ph.D. Thesis, hep-ph/9606474.

3. G. Sterman, Nucl. Phys. B281 (1987) 310.

4. N. Kidonakis, J. Smith, and R. Vogt, Phys. Rev. D 56 (1997) 1553; N. Kidonakis, in $Q C D$ 97, Nucl. Phys. B (Proc. Suppl.) 64 (1998) 402; N. Kidonakis and R. Vogt, Phys. Rev. D 59 (1999) 074014.

5. R. Meng, G.A. Schuler, J. Smith, and W.L. van Neerven, Nucl. Phys. B339 (1990) 325.

6. N. Kidonakis, E. Laenen, S. Moch, and R. Vogt, in preparation.

7. N. Kidonakis and J. Smith, Phys. Rev. D 51 (1995) 6092; hep-ph/9506253.

8. N. Kidonakis, G. Oderda, and G. Sterman, Nucl. Phys. B525 (1998) 299; B531 (1998) 365.

9. E. Laenen, G. Oderda, and G. Sterman, Phys. Lett. B 438 (1998) 173.

10. N. Kidonakis and J. Owens, in preparation.

11. L.E. Gordon and W. Vogelsang, Phys. Rev. D 48 (1993) 3136.

12. E706 Collaboration, L. Apanasevich et al., Phys. Rev. Lett. 81 (1998) 2642. 